\title{
EVALUATION OF ARGINASE ACTIVITY, NITRIC OXIDE AND OXIDATIVE STRESS STATUS IN SHEEP WITH CONTAGIOUS AGALACTIA
}

\author{
Basak HANEDAN $^{1 *}$, Akin KIRBAs ${ }^{1}$, Fatih Mehmet KANDEMIR ${ }^{2}$, Mustafa Sinan AKTAS ${ }^{1}$ \\ and Ahmet YILDIZ ${ }^{3}$ \\ ${ }^{1}$ Department of Internal Medicine, ${ }^{2}$ Department of Biochemistry and ${ }^{3}$ Department of Ani- \\ mal Science, Faculty of Veterinary Medicine, Ataturk University, 25170 Erzurum, Turkey
}

(Received 6 December 2016; accepted 4 April 2017)

It is known that inflammatory organ damages due to various agents, such as microorganisms including mycoplasmas, lead to oxidative stress. Nitric oxide (NO) functions as an antimicrobial agent, and arginase decreases proinflammatory cytokine release. There are very few studies on arginase activity, NO level and oxidative stress status in mycoplasmal infections. Therefore, the aim of this study was to evaluate erythrocyte arginase activity, plasma NO level and oxidative stress status in sheep with contagious agalactia. The study material consisted of 10 healthy sheep and 14 sheep with contagious agalactia characterised by mastitis, arthritis and keratoconjunctivitis. Erythrocyte arginase activity, plasma NO, malondialdehyde (MDA), total oxidant capacity (TOC) and total antioxidant capacity (TAC) levels were measured. Significant decreases in erythrocyte arginase activity and plasma TAC level $(\mathrm{P}<0.001)$, and significant increases in plasma NO, MDA and TOC levels $(\mathrm{P}<0.001)$ were found in the diseased sheep as compared with the healthy animals. This study suggests that contagious agalactia may cause oxidative stress due to increased plasma MDA and TOC levels and decreased plasma TAC levels, and that the decrease in erythrocyte arginase activity and increase in plasma NO level may contribute to the elimination of mycoplasmal agents causing contagious agalactia. oxide

Key words: Contagious agalactia, oxidative stress, arginase activity, nitric

Contagious agalactia occurs worldwide, especially in southern Europe and the Middle East, with significant economic losses (Scott, 2007). Mycoplasma agalactiae is a major causative agent responsible for contagious agalactia (CA) in sheep. The occurrence of clinical CA in sheep herds is often attributable to the entry of infected animals or to diminished herd immunity (Gomez-Martin et al., 2013). The causative agent is transmitted by direct contact, aerosol transmission,

*Corresponding author; E-mail: fbhanedan@gmail.com; Phone: 0090 (442) 231-7171; Fax: 0090 (442) 231-5563 
ingestion, and contaminated fomites. CA causes septicaemia, arthritis, mastitis, conjunctivitis, pneumonia, and abortion (Bergonier et al., 1997; Radostits et al., 2006; Kumar et al., 2014). The diagnosis of CA is made by culture, PCR and ELISA tests (Radostits et al., 2006). Antibiotic treatment reduces the severity of clinical signs but leads to a carrier state (Nicholas, 2002).

Mycoplasma agalactiae causes inflammatory organ damages, especially severe diffuse purulent mastitis, characterised by lymphoplasmacytic infiltration as shown by experimental infection in goats (Castro-Alonso et al., 2009). Immune reactions, in particular infections, may produce considerable amounts of oxidants (reactive oxygen species, ROS) released from inflammatory cells that target microorganisms and also cause tissue injury (Lykkesfeldt and Svendsen, 2007). In addition, inducible nitric oxide synthase (iNOS) is induced by lipopolysaccharide (LPS) and various cytokines (Wu and Morris, 1998), and it produces superoxide and nitric oxide (NO) (Lykkesfeldt and Svendsen, 2007). NO functions as an antimicrobial agent against intracellular pathogens (Chakravortty and Hensel, 2003). Arginase competes with nitric oxide synthase (NOS) for L-arginine (Wu and Morris, 1998). Arginase converts L-arginine to urea and ornithine (Mori, 2007). LPS and type 2 cytokines cause arginase I expression in macrophages, while type 1 cytokines induce NO production by iNOS. The polyamines produced in the arginase pathway decrease the release of proinflammatory cytokines (Das et al., 2010).

Antioxidants play a role in making oxidants harmless to cellular molecules. Enzymatic and nonenzymatic antioxidants protect cells from oxidants. Excess oxidants may damage the cells and induce programmed cell death (Lykkesfeldt and Svendsen, 2007).

It is recognised that inflammatory organ damages due to various agents, such as microorganisms including mycoplasmas, lead to oxidative stress. NO functions as an antimicrobial agent and arginase decreases the release of proinflammatory cytokines (Chakravortty and Hensel, 2003; Castro-Alonso et al., 2009; Das et al., 2010). There are very few studies on arginase activity, NO level and oxidative stress status in mycoplasmal infections. Therefore, the aim of this study was to evaluate erythrocyte arginase activity, plasma NO level and oxidative stress status in sheep with contagious agalactia.

\section{Materials and methods}

\section{Animals}

The study material consisted of 10 healthy sheep and 14 sheep with contagious agalactia characterised by mastitis, arthritis, and keratoconjunctivitis. The sheep were 1-2 years old and kept on a private farm in Erzurum province. They were examined for clinical signs and the diagnosis was made by ELISA test at 
the Veterinary Control and Research Institute. Blood samples were obtained from the jugular vein of healthy and diseased sheep and centrifuged at $3000 \times g$ for $10 \mathrm{~min}$. NO, malondialdehyde (MDA), total oxidant capacity (TOC) and total antioxidant capacity (TAC) levels were measured in the plasma while erythrocyte arginase activities were measured in erythrocytes.

The study was approved by the Ethics Committee of Ataturk University.

\section{Erythrocyte arginase activity analysis}

The spectrophotometric analysis of erythrocyte arginase activity was performed by the thiosemicarbazide-diacetyl monoxime-urea method (Geyer and Dabich, 1971). Diacetyl monoxime was hydrolysed to diacetyl and hydroxylamine via the effect of heat in an acidic medium. Diacetyl was converted to diazine, a compound of yellow colour, through condensation reaction with urea in acidic solution. Diazine formation is directly proportional to the amount of arginase. The formed yellow colour was stabilised by the use of thiosemicarbazide and $\mathrm{Fe}^{+2}$ (Kaplan, 1987). Haemoglobin was measured by the Drabkin method (Fairbanks and Klee, 1986).

\section{Plasma nitric oxide (NO) analysis}

An NO detection kit (Enzo Life Science) was used for measuring plasma total NO level. The plasma NO measurement is based on the enzymatic conversion of nitrate to nitrite and the colorimetric detection of nitrite, a coloured azo dye product of the Griess reaction.

\section{Plasma malondialdehyde (MDA) analysis}

Plasma MDA levels were measured spectrophotometrically using the method of Placer et al. (1966). In this method, $250 \mu \mathrm{l}$ of plasma and $2.25 \mathrm{ml}$ of colouring agent (a mix of three parts of trichloroacetic acid and one part of thiobarbituric acid) were mixed in the sample tube, and $250 \mu 1$ of normal saline and $2.25 \mathrm{ml}$ colouring reagent were mixed in the blank tube. The sample and blank tubes were heated in boiling water bath for $20 \mathrm{~min}$. Then the tubes were cooled, centrifuged at $2500 \mathrm{rpm}$ for $5 \mathrm{~min}$, and measurements were made at $532 \mathrm{~nm}$ against the blank.

\section{Plasma total oxidant capacity (TOC) analysis}

Plasma TOC levels were measured using a novel automated method developed by Erel (2005) (TOC assay kit, Rel Assay Diagnostic). Oxidants in the plasma sample convert the ferrous ion-O-dianisidine complex to ferric ion. The oxidation reaction is increased by glycerol molecules abundantly present in the reaction medium. The ferric ion forms a coloured complex with xylenol orange in an acidic medium. The colour intensity is measured spectrophometrically. 


\section{Plasma total antioxidant capacity (TAC) analysis}

The plasma TAC levels were measured using a novel automated method developed by Erel (2004) (TAC assay kit, Rel Assay Diagnostic). The oxidative reactions started by hydroxyl radicals in the reaction mixture are inhibited by the antioxidant components in the sample, and inhibition of the colour change and TOC are measured in the plasma sample.

\section{Statistical analysis}

Statistical analysis was performed to compare the diseased and the healthy group. The Kolmogorov-Smirnov test was used for the test of normality, followed by an independent samples $t$-test. $\mathrm{P}$ values less than 0.05 were considered significant.

\section{Results}

The diseased sheep had normal respiratory and pulse rates and their heart and lung auscultation findings were unchanged. Defects and vascularisation of the cornea were detected. The carpal and tarsal joints were slightly swollen. There was a significant loss of milk production, and milk samples from the udder of diseased sheep showed watery yellow or grey discolouration and clot formation. The diseased sheep revealed complications including mastitis, arthritis and keratoconjunctivitis specific for contagious agalactia. The diseased sheep herd was culled by the owner because of the positive ELISA test result and clinical findings specific for contagious agalactia.

A significant decrease in erythrocyte arginase activity and plasma TAC levels ( $\mathrm{P}<0.001)$ as well as a significant increase in plasma NO, MDA, and TOC levels $(\mathrm{P}<0.001)$ were determined in the diseased sheep having complications such as mastitis, arthritis and keratoconjunctivitis as compared with the healthy sheep (Table 1).

\section{Table 1}

Erythrocyte arginase activity, nitric oxide, malondialdehyde, total oxidant capacity and total antioxidant capacity levels in healthy and diseased sheep

\begin{tabular}{lcrc}
\hline Parameters & $\begin{array}{c}\text { Healthy sheep } \\
(\text { mean } \pm \text { SD) }\end{array}$ & $\begin{array}{c}\text { Diseased sheep } \\
(\text { mean } \pm \text { SD) }\end{array}$ & $P$ \\
\hline Arginase activity (U/g haemoglobin) & $54.57 \pm 5.22$ & $29.54 \pm 5.85$ & $<0.001$ \\
$\mathrm{NO}(\mu \mathrm{mol} / \mathrm{l})$ & $41.51 \pm 3.11$ & $50.06 \pm 3.03$ & $<0.001$ \\
$\mathrm{MDA}(\mathrm{nmol} / \mathrm{ml})$ & $3.72 \pm 0.24$ & $5.17 \pm 0.48$ & $<0.001$ \\
TOC $\left(\mu \mathrm{mol} \mathrm{H} \mathrm{O}_{2}\right.$ equivalent/l) & $1.62 \pm 0.06$ & $1.94 \pm 0.15$ & $<0.001$ \\
TAC $(\mathrm{mmol} \mathrm{Trolox} \mathrm{equivalent/l)}$ & $1.11 \pm 0.11$ & $0.89 \pm 0.10$ & $<0.001$ \\
\hline
\end{tabular}

NO: Nitric oxide; MDA: Malondialdehyde; TOC: Total oxidant capacity; TAC: Total antioxidant capacity 


\section{Discussion}

Sheep with contagious agalactia exhibited major clinical signs including severe mammary infection with loss of milk, watery yellow or grey discolouration of milk flow, lameness, carpal or tarsal joint swelling, and corneal vascularisation. Mycoplasma agalactiae can disseminate from the site of infection to various internal organs (Hedge et al., 2014). In this study, the diseased sheep revealed complications such as mastitis, arthritis and keratoconjunctivitis specific for contagious agalactia. The affected sheep herd was culled by the owner since persistent chronic infections occur in animals infected with contagious agalactia.

Chronic persistent infections can be associated with the ability of $M$. agalactiae to enter the host cells and to exit the cells to propagate in the extracellular environment through variable surface components by escaping the host immune cells and antibiotic action (Chopra-Dewasthaly et al., 2012; Hedge et al., 2014) and to produce peroxiredoxins which significantly contribute to their resistance against oxidative and nitrosative stress produced by the natural immune response (Jaeger et al., 2004).

Mycoplasma lipoproteins are potent immunogens (Chambaud et al., 1999). Serum antibody increase, lymphoplasmacytic inflammatory reaction and the increase in macrophage numbers indicate the humoral and cellular immune response against M. agalactiae (Castro-Alonso et al., 2009, 2010). Activation of macrophages reduces the number of mycobacterial organisms by producing ROS and reactive nitrogen intermediates (RNI) (Morris et al., 2013). In this study, a significant increase in MDA and TOC levels and a significant decrease in TAC levels were found in sheep with contagious agalactia compared with healthy sheep, in accordance with the findings of Kizll et al. (2007) who reported that a significant increase in MDA and a significant decrease in GSH-Px, GSH, vitamins $\mathrm{E}$ and $\mathrm{C}$ and $\beta$-carotene were found in goats infected with $M$. agalactiae compared with healthy goats. Thus, this study has suggested that oxidative stress can occur in sheep with contagious agalactia due to increased MDA and TOC levels as a marker of increased oxidants and decreased TAC levels as a marker of decreased antioxidants.

Reactive nitrogen intermediates are important antimicrobial molecules and induce more innate immunity than ROS against microbial infections. NO is an important member of RNIs, inhibiting the growth of mycobacterial organisms (MacMicking et al., 1997; Bogdan, 2001; Chan et al., 2001; Pieters, 2008; Morris et al., 2013).

iNOS is induced by LPS and type 1 cytokines such as interferon gamma (IFN $\gamma$ ), tumour necrosis factor alpha (TNF- $\alpha$ ), interleukin 1 (IL-1) and IL-2. The production of NO by iNOS is required for an efficient immune response killing the invading pathogens in macrophages (Das et al., 2010; Luiking et al., 2010). NO is also reported to be an effective antimicrobial agent against intracellular 
pathogens (Chakravortty and Hensel, 2003). If NO production is decreased and arginase 1 activity is increased in the macrophages, mycobacteria can evade the host immune response (Qualls et al., 2010).

Arginase can be produced in monocytes and macrophages (Mori, 2007). The production of arginase enzyme in macrophages is induced by LPS and type 2 cytokines such as IL-4, IL-13 and TGF- $\beta$. Type 2 cytokines inhibit iNOS and induce arginase activity (Das et al., 2010). Arginase activity provides tissue repair through polyamine synthesis (Shearer et al., 1997). On the other hand, arginase activity can contribute to the growth of bacterial pathogens (Das et al., 2010). In this study, a significant increase in plasma NO levels and a significant decrease in erythrocyte arginase activity were demonstrated in the diseased sheep compared with the healthy animals, suggesting that in sheep with contagious agalactia there is a response that attempts to kill $M$. agalactiae by increasing plasma NO levels and decreasing erythrocyte arginase activities.

In conclusion, this study suggests that contagious agalactia may cause oxidative stress, and that decreased erythrocyte arginase activity and increased plasma NO level may contribute to the elimination of mycoplasmal agents causing contagious agalactia. Future studies should be conducted to evaluate the antimicrobial efficacy of high NO production via nitric oxide donors as part of CA treatment.

\section{References}

Bergonier, D., Berthelot, X. and Poumarat, F. (1997): Contagious agalactia of small ruminants: Current knowledge concerning epidemiology, diagnosis and control. Rev. Sci. Tech. 16, $848-873$.

Bogdan, C. (2001): Nitric oxide and the immune response. Nat. Immunol. 2, 907-916.

Castro-Alonso, A., De la Fe, C., Espinosa de los Monteros, A., Rodriguez, F., Andrada, M., Poveda, J. B. and Herraez, P. (2010): Chronological and immunohistochemical characterization of the mammary immunoinflammatory response in experimental caprine contagious agalactia. Vet. Immunol. Immunopathol. 136, 43-54.

Castro-Alonso, A., Rodriguez, F., De la Fe, C., Espinosa de los Monteros, A., Poveda, J. B., Andrada, M. and Herraez, P. (2009): Correlating the immune response with the clinicalpathological course of persistent mastitis experimentally induced by Mycoplasma agalactiae in dairy goats. Res. Vet. Sci. 86, 274-280.

Chakravortty, D. and Hensel, M. (2003): Inducible nitric oxide synthase and control of intracellular bacterial pathogens. Microbes Infect. 5, 621-627.

Chambaud, I., Wroblewski, H. and Blanchard, A. (1999): Interactions between mycoplasma lipoproteins and the host immune system. Trends Microbiol. 7, 493-499.

Chan, E. D., Chan, J. and Schluger, N. W. (2001): What is the role of nitric oxide in murine and human host defense against tuberculosis? Current knowledge. Am. J. Respir. Cell Mol. Biol. 25, 606-612.

Chopra-Dewasthaly, R., Baumgartner, M., Gamper, E., Innerebner, C., Zimmermann, M., Schilcher, F., Tichy, A., Winter, P., Jechlinger, W., Rosengarten, R. and Spergser, J. (2012): Role of Vpma phase variation in Mycobacteria agalactiae pathogenesis. FEMS Immunol. Med. Microbiol. 66, 307-322. 
Das, P., Lahiri, A., Lahiri, A. and Chakravortty, D. (2010): Modulation of the arginase pathway in the context of microbial pathogenesis: A metabolic enzyme moonlighting as an immune modulator. PLoS Pathog. 6, e1000899.

Erel, O. (2004): A novel automated direct measurement method for total antioxidant capacity using a new generation, more stable ABTS radical cation. Clin. Biochem. 37, 277-285.

Erel, O. (2005): A new automated colorimetric method for measuring total oxidant status. Clin. Biochem. 38, 1103-1111.

Fairbanks, V. F. and Klee, G. G. (1986): Biochemical aspects of hematology. In: Tiets, N. W. (ed.). Textbook of Clinical Chemistry. W. B. Saunders Company, Philadelphia. pp. 1532-1534.

Geyer, J. W. and Dabich, D. (1971): Rapid method for determination of arginase activity in tissue homogenates. Anal. Biochem. 39, 412-417.

Gomez-Martin, A., Amores, J., Paterna, A. and De la Fe, C. (2013): Contagious agalactia due to Mycoplasma spp. in small dairy ruminants: Epidemiology and prospects for diagnosis and control. Vet. J. 198, 48-56.

Hedge, S., Hedge, S., Spergser, J., Brunthaler, R., Rosengarten, R. and Chopra-Dewasthaly, R. (2014): In vitro and in vivo cell invasion and systemic spreading of Mycoplasma agalactiae in the sheep infection model. Int. J. Med. Microbiol. 304, 1024-1031.

Jaeger, T., Budde, H., Flohe, L., Menge, U., Singh, M., Trujillo, M. and Radi, R. (2004): Multiple thioredoxin-mediated routes to detoxify hydroperoxides in Mycobacterium tuberculosis. Arch. Biochem. Biophys. 423, 182-191.

Kaplan, L. A. (1987): Urea. In: Pesce, A. J. and Kaplan, L. A. (eds) Methods in Clinical Chemistry. The CV Mosby Company, Toronto. pp. 22-27.

Kızıl, O., Ozdemir, H., Karahan, M. and Kızıl, M. (2007): Oxidative stress and alterations of antioxidant status in goats naturally infected with Mycoplasma agalactiae. Rev. Med. Vet. 158, 326-330.

Kumar, A., Rahal, A., Chakraborty, S., Verma, A. K. and Dhama, K. (2014): Mycoplasma agalactiae, an etiological agent of contagious agalactia in small ruminants: A review. Vet. Med. Int. 2014, 286752. doi: 10.1155/2014/286752. Epub 2014 July 3.

Luiking, Y. C., Engelen, M. P. and Deutz, N. E. (2010): Regulation of nitric oxide production in health and disease. Curr. Opin. Clin. Nutr. Metab. Care 13, 97-104.

Lykkesfeldt, J. and Svendsen, O. (2007): Oxidants and antioxidants in disease: Oxidative stress in farm animals. Vet. J. 173, 502-511.

MacMicking, J., Xie, Q. W. and Nathan, C. (1997): Nitric oxide and macrophage function. Annu. Rev. Immunol. 15, 323-350.

Mori, M. (2007): Regulation of nitric oxide synthesis and apoptosis by arginase and arginine recycling. J. Nutr. 137, 1616S-1620S.

Morris, D., Khurasany, M., Nguyen, T., Kim, J., Guilford, F., Mehta, R., Gray, D., Saviola, B. and Venketaraman, V. (2013): Glutathione and infection. Biochim. Biophys. Acta 1830, 3329-3349.

Nicholas, R. A. J. (2002): Improvements in the diagnosis and control of diseases of small ruminants caused by mycoplasmas. Small Rum. Res. 45, 145-149.

Pieters, J. (2008): Mycobacterium tuberculosis and the macrophage: maintaining a balance. Cell Host Microbe 3, 399-407.

Placer, Z. A., Cushman, L. and Johnson, B. C. (1966): Estimation of products of lipid peroxidation (malonyl dialdehyde) in biological fluids. Anal. Biochem. 16, 359-364.

Qualls, J. E., Neale, G., Smith, A. M., Koo, M. S., DeFreitas A. A., Zhang, H., Kaplan, G., Watowich, S. S. and Murray, P. J. (2010): Arginine usage in mycobacteria-infected macrophages depends on autocrine-paracrine cytokine signaling. Sci. Signal. 3, 135, ra62. doi: 10.1126/scisignal.2000955.

Radostits, O. M., Gay, C. C., Hinchcliff, K. W. and Constable, P. D. (2006): Veterinary Medicine. A Textbook of the Diseases of Cattle, Sheep, Goats, Pigs and Horses. 10th edition. Saunders Elsevier, Edinburgh, London, New York. 
Scott, P. R. (2007): Contagious Agalactia. Sheep Medicine. Manson Publishing/The Veterinary Press.

Shearer, J. D., Richards, J. R., Mills, C. D. and Caldwell, M. D. (1997): Differential regulation of macrophage arginine metabolism: a proposed role in wound healing. Am. J. Physiol. 272, E181-E190.

Wu, G. and Morris, S. M. Jr. (1998): Arginine metabolism: nitric oxide and beyond. Biochem. J. 336, $1-17$. 